\title{
La ingeniería industrial y el mercado verde internacional
}

\author{
Jaramillo Camila ${ }^{1}$ \\ ORCID: 0000-0002-3522-5328 \\ Universidad de las Américas \\ Quito-Ecuador
}

\author{
Patiño Ariana ${ }^{2}$ \\ ORCID: 0000-0001-5525-5308 \\ Universidad de las Américas \\ Quito-Ecuador
}

Recibido (09/11/20), Aceptado (18/11/20)

\begin{abstract}
Resumen: Este ensayo tiene como objetivo enfatizar las alternativas que se están tomando a nivel global para disminuir el impacto medio ambiental que tiene la actividad humana. Se desarrollarán puntos y conceptos claves en cuanto al mercado verde internacional, las empresas socialmente responsables (ESR) y la importancia de estos en la sociedad y economía actual. Dichos conceptos han ido adquiriendo mayor fuerza en la última década debido a los nuevos consumidores, más preocupados y empáticos con el medio ambiente y la comunidad, y a las empresas que buscan satisfacer las necesidades de estos consumidores siendo socialmente responsables sin dejar de ser rentables.
\end{abstract}

Palabras Clave: sociedad, economía, medio ambiente.

\section{International green market \& Industrial Engineering}

\begin{abstract}
This essay aims to emphasize the alternatives that are being taken globally to reduce the environmental impact of human activity. Key points and concepts will be developed regarding the international green market, socially responsible companies (SRC) and the relevance of these in modern society and economy. These concepts have been gaining strength in the last decade due to new consumers, who are more concerned and empathic with the environment and the community, and to companies that pursue to satisfy the needs of these consumers while being socially responsible while remaining profitable.
\end{abstract}

Keywords: society, economy, environment. 


\section{I.INTRODUCCIÓN}

El calentamiento global pasó de ser un tema netamente altruista promovido por grupos ecologistas

a escala mundial, a convertirse en un factor determinante en las políticas del mercado de todos los sectores económicos [1]. En este contexto surge el concepto de mercados verdes, que constituyen nuevos mercados de productos y servicios eco amigables, y de aquellos nuevos productos que surgen a partir del ecodiseño y del aprovechamiento sostenible del medio ambiente. Además, se recalca la trascendencia de los llamados consumidores verdes, los cuales comprenden la importancia y, los beneficios sociales y ambientales del consumo de productos biodegradables y eco amigables. Así el mercado verde constituye una alternativa sustentable al mercado tradicional, en el cual se fabrican cada vez más productos constituidos por componentes dañinos para el medio ambiente lo que los hace cada vez menos preferidos por los llamados "nuevos" consumidores [2].

Además, el mercado verde conlleva la creación de nuevas empresas enfocadas en la responsabilidad social hacia sus colaboradores, la sociedad y el medio ambiente. Estas establecen una nueva visión de empresa, en donde se busca lograr un éxito no solamente basado en los ingresos económicos y en el cumplimiento de las leyes, sino que también en la sustentabilidad de su marca y productos asegurando así su permanencia dentro del mercado y la fidelidad de sus clientes. Este tipo de empresas generan un "plus" en sus productos, al no constituir una amenaza para el medio ambiente y ser beneficiosos para la salud, que llama la atención de los consumidores y las diferencias dentro de sus mercados.

En este trabajo se expondrá el impacto de la creación de nuevos mercados verdes, el comportamiento del consumidor verde y los beneficios de la creación de nuevas empresas enfocadas en la responsabilidad social.

\section{II.DESARROLLO}

El mercado verde es una la red de comercialización de los productos obtenidos a partir de prácticas

agrícolas y pecuarias más amigables con el medio ambiente, con una tácita eliminación o disminución en el uso de insumos químicos, de acuerdo con un uso mucho más racional de estos. Sin embargo, el mercado de lo verde va mucho más allá [1], ya que los productos fabricados a partir de materiales naturales, productos orgánicos, productos fabricados a partir del ecodiseño, y productos obtenidos a partir de energías y tecnologías sostenibles también entran dentro de este tipo de mercados.

El mercado verde europeo es mucho más amplio y diverso comparado con el latinoamericano, esto debido al comportamiento de sus consumidores y a la cultura verde que está ampliamente fomentada dentro de estos países. Según una encuesta realizada a los consumidores españoles y alemanes [3] respecto a los factores que los impulsan a realizar una compra ecológica, se concluyó que la principal motivación de encuestados españoles para comprar productos alimenticios verdes es el beneficio que genera el consumo de estos alimentos para la salud, además de la calidad de estos. Los consumidores alemanes también piensan lo mismo, pero además tienen en cuenta el beneficio de la protección medioambiental que caracteriza a este tipo de productos.

Cuando se habla de mercado verde es también es importante mencionar al consumidor, en muchos casos llamado consumidor verde, ya que como se ha evidenciado en muchos mercados europeos el aumento del consumo de artículos biodegradables y eco amigables ha resultado en el surgimiento de nuevas empresas que se dedican a fabricar este tipo de productos. Además, esto ha incrementado el número de estudios acerca de nuevos materiales que puedan sustituir el uso de plástico y otros materiales de alto consumo que debido a su origen y ciclo de vida afectan directamente al bienestar del medio ambiente.

Así se denota la importancia del consumidor dentro de la creación de nuevos mercados verdes, debido a que la mayoría de las empresas exitosas estudian constantemente al mercado y siempre tratan de innovar de acuerdo con la demanda cambiante de los consumidores; entonces se podría decir que si la mayoría de las personas empieza a consumir productos amigables con el ambiente se empezarán a crear nuevos mercados con productos que cada vez son más innovadores y no contaminantes. Pero hay que tomar en cuenta que muchas personas están acostumbradas a comprar productos desechables, de plástico y de papel, entre otros; debido a la facilidad de adquisición de estos. Además, otra razón por la cual en muchos países los productos ecológicos no son populares o de consumo masivo es que existe un desconocimiento general de la existencia de estos. También existe la creencia de que este tipo de productos tienen un precio alto comparado con otros que son de tipo contaminante, lo cual en algunas ocasiones no es cierto, ya que en la actualidad la mayoría de las empresas buscan ser competitivas dentro de sus mercados y siempre tratan de ofrecer precios que se ajusten a su demanda de consumidores. Por otro lado, se podrían considerar a los productos eco-amigables como una inversión, puesto que a largo plazo resultan más 
económicos que el estar adquiriendo constantemente productos de un solo uso.

Las actividades humanas están afectando de forma profunda a la mayor parte de los procesos que determinan, globalmente, el funcionamiento de la biosfera y se estima que durante las próximas décadas la humanidad necesitará rediseñar sus patrones actuales de crecimiento y desarrollo si no quiere acabar enfrentándose a un colapso ecológico y social cuyas consecuencias podrían ser dramáticas [4]. Es por lo que se reitera la importancia de la creación de nuevos mercados verdes y de que las empresas lleven la responsabilidad social mucho más allá del cumplimiento de las leyes; se necesita un cambio de mentalidad en las personas para que se empiece a concientizar más sobre la importancia del medio ambiente y sobre los efectos negativos que el consumismo, la generación de basura y las industrias están teniendo sobre el planeta.

En este contexto se puede hablar de la importancia de la educación ambiental en la sociedad; las escuelas, colegios y universidades deberían fomentar este tipo de educación frecuentemente, pero es primordial que esta se ponga en práctica y no solamente se quede en conceptos y opiniones, ya que en muchas ocasiones las personas son conscientes de la contaminación que generan, pero no actúan de ninguna manera para evitarla o disminuirla. De esta manera se recalca la importancia de poner en práctica todos los conceptos y actividades aprendidas no solamente en las instituciones educativas, sino que también en casa, para así crear una cultura de responsabilidad social dentro de la sociedad.

En la actualidad, los consumidores han ido tomado consciencia de la grave situación ambiental y social en la que se encuentra el planeta y por ello se han inclinado hacia el consumo de productos o servicios de empresas socialmente responsables, pues reúnen condiciones de sustentabilidad ambiental al no contaminar, cuidar y aprovechar al máximo cada recurso e insumo evitando los desperdicios mediante la optimización de sus procesos logrados mediante investigación e innovación, desarrollando a su vez mejores productos. También, buscan el crecimiento de la economía local y beneficios para la comunidad más allá del generar dinero como ocurre con la mayoría de las empresas tradicionales, todo esto manteniéndose competitivas y rentables.

La responsabilidad social empresarial se relaciona con la sociedad en todos los horizontes, pues abarca a todos los stakeholders y debe tener una gestión estratégica adecuada, con objetivos y límites claramente establecidos para evitar la generación de altos costos, obtener beneficios y aceptación por parte del medio. Estas empresas son una inversión que genera resultados positivos a largo plazo y facilita la aceptabilidad en el mercado debido a las ventajas competitivas que generan mejora en la productividad, pertenencia a la empresa, mejor entorno laboral, reconocimiento del nombre de la empresa, entre otros aspectos positivos [5].

Las empresas socialmente responsables constituyen organizaciones que toman en cuenta tres aspectos fundamentales dentro de su modelo de negocio, la sociedad, la economía y el medio ambiente. En el ámbito económico buscan un beneficio monetario tomando en cuenta las consecuencias y/o los efectos negativos de sus actividades productivas o de servicio. En el ámbito social su objetivo es reforzar las relaciones con sus colaboradores y enfocarse en su beneficio ya que son conscientes de la importancia de estos para la organización, además se preocupan por el bienestar de la comunidad y ofrecen ayudas como donaciones y proyectos de beneficio social. Pero también son conscientes del impacto ambiental de sus procesos por lo que estos están enfocados hacia la sostenibilidad y sustentabilidad ambiental.

El término responsabilidad social de las empresas hace referencia al hecho de que estas buscan logar un éxito comercial integrando consideraciones sociales y medioambientales en las actividades de la empresa. En otras palabras, satisfacer la demanda de los clientes y gestionar, al mismo tiempo, las expectativas de otras partes interesadas: los trabajadores, proveedores y la comunidad de su entorno, además de contribuir de forma positiva a la sociedad gestionando el impacto medioambiental de la empresa [2]. Este tipo de empresas conllevan la responsabilidad social más allá del cumplimiento de las leyes, ya que uno de sus objetivos primordiales es el de crear nuevos mercados en donde se fomente el consumo responsable además de asegurarse del bienestar de la comunidad y se sus colaboradores.

Así el rol del Ingeniero Industrial dentro de las empresas socialmente responsables es esencial, debido a que el trabajo de los mismos dentro de las empresas manufactureras y de servicio es el de optimizar y, gestionar los procesos y procedimientos de una empresa tomando en cuenta todos los componentes de los mismos, así el Ingeniero Industrial tiene el deber de asegurarse de que la reingeniería de los procesos de las empresas se ejecute de manera que ninguna actividad industrial repercuta en un efecto negativo sobre la empresa; teniendo en cuenta los aspectos económicos, sociales y ambientales que constituyen a este tipo de empresas. 


\section{III.CONCLUSIONES}

A.El daño ambiental generado por las actividades humanas ha puesto en contexto las prioridades productivas, consumistas y comerciales que se han desarrollado a lo largo de los años y por ello se están generando diferentes actividades de cambio para frenar los daños mencionados. La mentalidad de las personas está cambiando pues está tomando un enfoque menos egoísta en cuanto al impacto del consumismo. Sin embargo, hay un gran porcentaje de personas que eligen la comodidad sobre el cuidado ambiental y por ello hay que seguir ejerciendo cambios tanto consumidores como productores.

B.Varias empresas han observado las problemáticas aludidas anteriormente y el cambio en las necesidades de los consumidores, por ello han surgido los mercados verdes y las empresas socialmente responsables, pero cabe recalcar que no son una solución única y perfecta para solucionar todo el daño ambiental generado hasta ahora, sin embargo, es una buena manera de incentivar un pensamiento más eco amigable en aquellos que no realizan estas prácticas.

C.Lo más importante para desarrollar todas estas acciones de cambio es hacerlo de manera ética, desinteresada y adquiriendo todo el conocimiento posible para evitar caer en el abuso de estos términos para beneficio propio, puesto que lo más importante es contribuir a la sociedad, mejorar como comunidad y evitar la destrucción del planeta.

\section{REFERENCIAS}

[1]W. Olaya González y L. A. Gómez Rodríguez, «¿Qué tan verde es tu mercado?,» Signo y Pensamiento, vol. XXX, no 58, pp. 314-324, 2011.

[2]C. González Rodríguez, «Empresas socialmente responsables y mercado verde internacional,» Economía Informa, $n^{\circ} 366$, pp. 59-78, 2011.

[3]R. Nikogosyan Sarkisova, «El auge del sector ecológico en europa: un estudio empírico del comportamiento del consumidor verde en Alemania y España,» Universidad de León, León, 2019.

[4]M. Aguado Caso, «Vivir bien en un planeta finito. Una mirada socio-ecológica al concepto de bienestar humano,» Universidad Autónoma de Madrid, Madrid, 2016.

[5]S. S. d. Valle, «La responsabilidad social empresarial: gestión estratégica para la supervivencia de las empresas,» Dimensión Empresarial, vol. 9, nº 2, pp. 6-15, 2011.

\section{RESUMEN CURRICULAR}

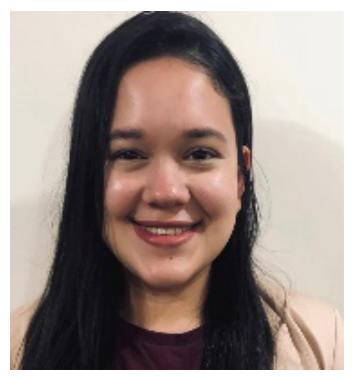

Ariana Patiño, Estudiante de séptimo semestre de la carrera de Ingeniería Industrial en la Universidad de las Américas.

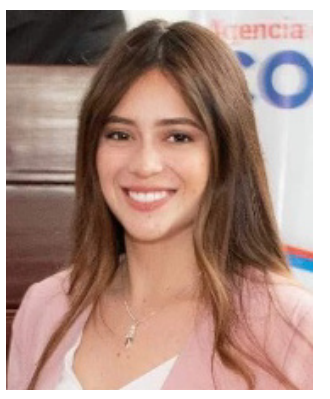

Camila Jaramillo, Estudiante de séptimo semestre de la carrera de Ingeniería Industrial en la Universidad de las Américas. 